\title{
Construction and analysis of the production function for complex structured productions
}

\author{
Mikhail Kharitonov ${ }^{1}$ \\ ${ }^{1}$ Mathematics and IT Department, Volgograd State University, 400062 Volgograd, Russia
}

\begin{abstract}
The production function of the complex structured productions is represented as a superposition of Leontief's production functions corresponding to each of the modules. The optimization problem is reduced to a linear programming problem with a parameter, which describes the operational structure of the core.
\end{abstract}

\section{Introduction}

The high efficiency of modern organizations in a competitive environment, within globalization processes, information, technical and technological innovations depends on the constant high adaptability, ie continuous organizational change. The problem of organizational (structural) changes in the past decades has been widely discussed in many works on management and organizational theory. The most notable works are Adizes I. [1], Blake R.R. and Mouton J.S. [2], Champy J.[3], Grant R.M Rami S., Krishnan R. [4], Kauffman S. [5], Mincberg G. [6], Watzlawick P., Weakland J., Fisch R [7], Tran Q. and Tian Y. [8], Weick K.Е. и Quinn R.E. [9], Sah R.K. [10], Hart O. [11]. At the same time against the backdrop of the many informal theories and models of adaptation and development of organizations there is a very few relevant conceptual mathematical models (Barnett W.P. and Carroll G.R. [12], Novikov D.A. [13, 14], Voronin A.A. and Mishin S.P. [15, 16], Gubko M.V. [17-18]).

To date, this area doesn't have yet a more or less general conceptual and algorithmic mathematical apparatus, and the available results are fragmentary. Various grounds for the classification of an organization's management problems produce different approaches to the problems of optimization of the organizational structure. One of these grounds is the time interval separating the management of the operation and management of changes. Optimization of structure traditionally is in the last set of administrative problems.

It appears impossible to construct large-horizon control models for an organizational system (OS) based on the system of control mechanisms [13] by disregarding its structural and functional variability. Actually, the variability of internal and external relations, short-, medium- and long-term goals and criteria of OS development stimulates further generalization of the models of the basic mechanisms [13] and the notion of a control mechanism. Such generalization lies in embedding (in addition to decision making methods) a complex of organizational structures, norms and parameters of an OS, that vary on a large control horizon according to the actual and desired dynamics of the OS as the result of implementing a sequence of simple and complex control mechanisms [13]. These generalized control mechanisms represent systems with a complicated structure, whose subsystems and elements are generalized, complex or simple mechanisms. Therefore, the optimality of each mechanism is defined within the framework of the optimization problem of the whole system.

As a matter of fact, this concept coincides with the concept of an organizational system pioneered by $\mathrm{H}$. Minzberg [6]. And so, the notions of an organizational structure and a generalized control mechanism act as synonyms; accordingly, Minzberg's structural configurations can be called optimal generalized control mechanisms. Consequently, the structural and organizational changes in an OS can be treated as transitions between conditionally optimal structural configurations or generalized control mechanisms, with separation of different paths (adaptation, development, etc.) depending on necessary attributes. It seems feasible to relate the development of an OS to the interaction and development of its generalized control mechanisms.

Researchers identify several stages of generalized mechanisms synthesis, i.e., modeling of the structural dynamics and dynamic structural optimization of an OS. These stages include construction of a family of corresponding dynamic equations and an efficiency criterion (in the structure-dependent form), description of the space of structural states, correct formulation of the dynamics "principles," as well as elaboration of efficient optimization algorithms. Among first fruitful steps in this direction, we refer to the publications [1518], where the authors studied the problems of static and dynamic optimization of control hierarchies in OSs with the efficiency criterion in the form of a structurallydependent cost function. Generalized control

\footnotetext{
Corresponding author: kharitonov@volsu.ru
} 
mechanisms synthesis calls for associating these models described by the structurally-dependent production function (PF) of an OS with game-theoretic models of hierarchical control. The afore-said motivates designing a certain system of multiparametric dynamic models of optimization and control, that form an hierarchical structure of generalized control mechanisms in an OS.

This paper presents an optimization model for the operating core of an organization; following the terminology of Minzberg [6], we comprehend such core as a set of structural units of an OS directly implementing the basic production and auxiliary production (support facilities including personnel and equipment). The structure of the operating core is described by a special oriented graph with a variable number of layers. Here nodes indicate the basic technological module and the modules of support facilities, whereas edges stand for the inputs and outputs of the technological modules. The goal function has the structure-dependent form as the superposition of Leontief production functions corresponding to each of the modules.

Under given inputs (production factors) and structure of the operating core, the value of the production function represents the solution to a linear programming problem.

Increasing the number of support facilities, on the one hand, guarantees stable production output in the case of violating optimal proportions among production factors. On the other hand, such initiative causes higher prime costs. An alternative method to complicate the structure of the operating core consists in purchasing missing production factors on a market (if available). This approach appears most efficient in economies with a highly developed institutional environment. As a result, the structure of an OS gets simplified and special outsourcing organizations come into service. The latter provide support facilities, as well as implement traditional functions of an OS. Economies with a low level of institutional environment demonstrate a totally different tendency - vertical integration by absorption of related and support facilities. However, the model suggested in this paper does not explore the optimal balance problem of these tendencies. Here structural optimization of the operating core lies in seeking for the minimal number of its layers, which ensures the maximal value of the production function under given inputs (production factors). In other words, further increase in the number of such layers would not raise this value.

To tackle the problem, we develop a certain algorithm for automatic construction of the basic equations for each number of layers. Finally, the optimization problem is solved numerically for a wide range of the model variables and parameters.

In [19-20] presented some models of static and dynamic optimization of support structure of OS operating core. In this paper, we present a synthesis of model for control of support structure of OC OS with structure-dependent PF as a superposition of elementary Leontief PF as in [19-20].

\section{Problem of support structure optimization}

The model of a structure-dependent production function (PF) constructed in this paper bases on the models [21]. As the elementary PF of each structural element of the operating core with invariable technology (a simple transformer-ST), we involve the Leontief PF,

$$
f=k \min \left(\frac{f_{1}}{a_{1}}, \frac{f_{2}}{a_{2}}, \ldots, \frac{f_{m}}{a_{m}}\right),
$$

where $f_{i}$ indicate the values of arguments (transformation factors of production); $a_{1}, \ldots, a_{m}$ mean the technological coefficients; and $k$ is the normalizing factor.

Stable interfactor disproportions or fluctuations in transformation factors appreciably reduce the value of the PF of an ST (in comparison with its maximum). Within an OS, the flexibility of the PF with respect to its arguments is guaranteed through constructing a vertical structure of support facilities that produce the missing elements of transformation factors for the maximal efficiency of the basic ST. Therefore, the structure of factor production flows in the operating core of an OS can be expressed as a complex transformer (CT) - a multilevel structure of STs, where outputs of some STs represent inputs of the others. The structure of a CT acquires the form of an oriented graph, whose nodes answer for STs and edges correspond to factor flows. The last node is responsible for the basic production, whereas the rest nodes relate to auxiliary production (support facilities). The original production factors are the inputs of all STs, and the last (basic) ST outputs the product of the OS.

Consider a CT with the production factors $N, R^{l} N$, $R^{2} N, \ldots, R^{m-1} N\left(R^{I} N, R^{2} N, \ldots, R^{m-1} N\right.$ are the elements of the factors $R^{l}, R^{2}, \ldots, R^{m-1}$ respectively, that produce the missing element of the factor $N$ ). It is feasible to produce the element of the factor $N$ via $n$ serial STs (see Figure 1).

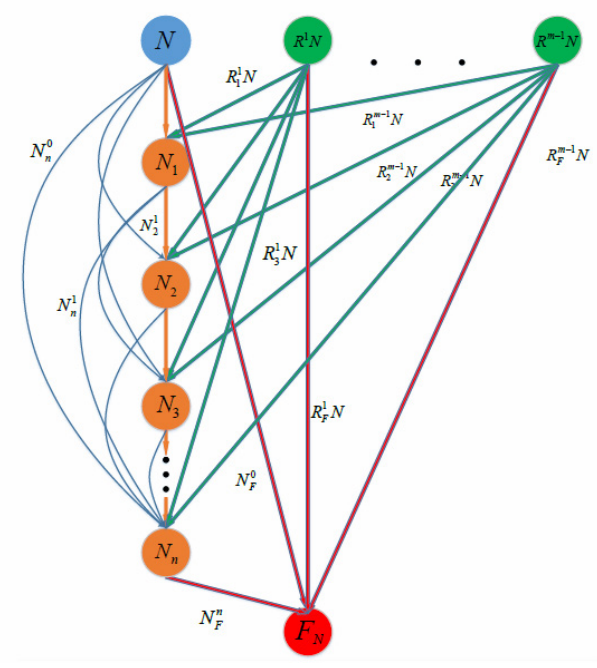

Fig 1. A complex transformer producing the element of the factor $N$ 
The PF of this CT satisfies the following system of equations:

$$
\begin{gathered}
\sum_{i=1}^{n} N_{i}^{0}+N_{F}^{0}=N, \sum_{i=1}^{n} R_{i}^{j} N+R_{F}^{j} N=R^{j} N, j=\overline{1, m-1} . \\
f_{1}=S_{a_{1}^{i}} \min \left(\frac{N_{1}^{0}}{a_{1}^{0}}, \frac{R_{1}^{1} N}{a_{1}^{1}}, \ldots, \frac{R_{1}^{m-1} N}{a_{1}^{m-1}}\right), S_{a_{1}^{i}}=\sum_{i=0}^{m-1} a_{1}^{i}, \\
f_{2}=S_{a_{2}^{i}} \min \left(\frac{f_{1}+N_{2}^{0}}{a_{1}^{0}}, \frac{R_{2}^{1} N}{a_{2}^{1}}, \ldots, \frac{R_{2}^{m-1} N}{a_{2}^{m-1}}\right), \\
f_{k}=S_{a_{k}^{i}} \min \left(\frac{1}{a_{k}^{0}}\left(f_{k-1}+\sum_{i=1}^{k-2} N_{k}^{i}+N_{k}^{0}\right), \frac{R_{k}^{1} N}{a_{k}^{1}}, \ldots, \frac{R_{k}^{m-1} N}{a_{k}^{m-1}}\right), \\
k=\overline{3, n} . \quad \\
F_{N}=S_{A^{i}} \min \left(\frac{1}{A^{0}}\left(f_{n}+\sum_{i=1}^{n-2} N_{n}^{i}+N_{F}^{0}\right), \frac{R_{F}^{1} N}{A^{1}}, \ldots, \frac{R_{F}^{m-1} N}{A^{m-1}}\right) .
\end{gathered}
$$

Take the variables of the system (1)-(2) and compile the factor flow vector of the CT:

$$
\boldsymbol{\varphi}(n, m)=\left(\left\{\left\{N_{i}^{j}\right\}_{i=j+1}^{n}\right\}_{j=0}^{n-1},\left\{\left\{R_{i}^{l}\right\}_{i=1}^{n}\right\}_{l=1}^{m-1},\left\{N_{F}^{i}\right\}_{i=1}^{n}, \varphi_{F}\right) .
$$

Under fixed $N, R^{l} N, R^{2} N, \ldots, R^{m-1} N$ the optimization problem for the PF of the CT (1)-(2) with variable structure takes the form

$$
F_{N}=S_{A^{i}} \min \left(\frac{f_{n}+\sum_{i=1}^{n-2} N_{n}^{i}+N_{F}^{0}}{A^{0}}, \frac{R_{F}^{1} N}{A^{1}}, \ldots, \frac{R_{F}^{m-1} N}{A^{m-1}}\right) \rightarrow \max _{n, \boldsymbol{\varphi}(n, m)} .
$$

\section{Analytical Estimating}

Let's make some statements on a possible estimation of the values of PF (3) for different values of technological coefficients, inter-factors imbalances and complexity of the CT structure.

Statement 1. For $m$-factor CT with positive technological coefficients $a_{i}^{j}, A^{j}, i=\overline{1, n}, j=\overline{0, m-1}$, and proportions between entries

$$
\frac{N}{R^{i} N}=x, \quad \frac{R^{j} N}{R^{i} N}=1, \quad x<1, \quad i, j=\overline{1, m-1}
$$

the solution of the optimization problem (3) has the following upper bound:

$$
F_{m}(n, x) \leq\left\{\begin{array}{l}
\frac{S_{A^{i}}}{A^{0}} \prod_{j=0}^{n} \frac{S_{a_{j}^{i}}}{a_{0}^{j}} x, n \leq n^{*} \\
x+m-1, n>n^{*}
\end{array},\right.
$$

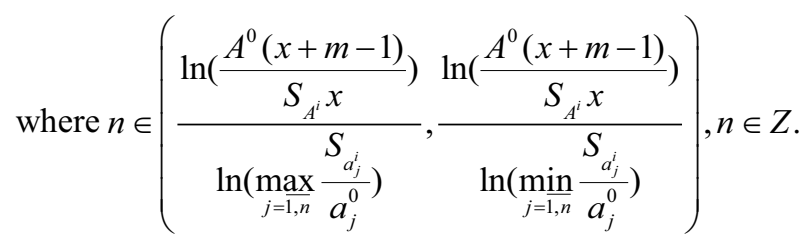

Statement 2. For $m$-factor CT with technological coefficients $a_{i}^{j}=1, A^{j}=1, i=\overline{1, n}, j=\overline{0, m-1}$, and proportions between entries

$$
\frac{N}{R^{i} N}=x, \quad \frac{R^{j} N}{R^{i} N}=1, \quad x<1, \quad i, j=\overline{1, m-1}
$$

the solution of the optimization problem (3) is

$$
F_{m}(n, x)=\left\{\begin{array}{l}
m^{n+1} x, n \leq n^{*} \\
x+m-1, n>n^{*}
\end{array}, n^{*}=\left\lfloor\frac{\ln \frac{m-1+x}{x}}{\ln m}\right\rfloor .\right.
$$

Statement 3. For $m$-factor CT with technological coefficients $a_{i}^{j}=1, A^{j}=1, i=\overline{1, n}, j=\overline{0, m-1}$, and proportions between entries

$$
\frac{R^{i} N}{N}=x, \quad \frac{R^{j} N}{R^{i} N}=1, \quad x<1, \quad i, j=\overline{1, m-1}
$$

the solution of the optimization problem (3) is $F_{m}(x, n)=m x$.

\section{Analytical Estimating}

Note that the problem (3) is equivalent to the following linear programming (LP) problem [21]:

$$
\begin{aligned}
& F_{N} \rightarrow \max _{n, \varphi_{N}},-\frac{S_{A^{i}}}{A^{0}}\left(f_{n}+\sum_{i=1}^{n-2} N_{n}^{i}+N_{F}^{0}\right)+F_{N} \leq 0, \\
& -\frac{S_{A^{i}}}{A^{j}} R_{F}^{j} N+F_{N} \leq 0, j=\overline{1, m-1 .}
\end{aligned}
$$

Similarly, the problem (1)-(3) gets reduced to the parametric LP problem

$$
\begin{aligned}
& F=\varphi_{F} \rightarrow \max _{\varphi(n, m), n}, \\
& \sum_{j=1}^{p(n, m)} \mathbf{M}(n, m)_{i j} \boldsymbol{\varphi}(n, m)_{j} \leq \boldsymbol{\beta}_{i}, i=\overline{1, u(n, m)}, \\
& \boldsymbol{\beta}=\left(N, R^{1}, R^{2}, \ldots, R^{m-1}, 0, \ldots, 0\right)^{T} .
\end{aligned}
$$

Here $p(n, m)$ means the number of variables and $u(n, m)$ indicates the number of inequalities; these quantities are defined by the formulas

$$
p(n, m)=\frac{n(n+2 m+3)}{2}, \quad u(n, m)=m(n+2) .
$$

Denote by $\varphi_{F}(n)$ the solution to the problem (4). We believe that the optimal structure of the CT described by the system (1)-(2) is the quantity $n^{*}=\min _{n}\left(\arg \max _{n} \varphi_{F}^{*}\right)$.

Set $\lambda=F /\left(N+R^{l} N+R^{2} N+\ldots+R^{m-1} N\right)$. The external parameters of the problem (4) comprise the initial interfactor proportions $N: R^{l} N: R^{2} N: \ldots: R^{m-1} N$ and the proportions of the technological coefficients. For each $\mathrm{CT}$, the quantities technological coefficients are assumed given. Numerical experiments employ random realizations of these parameters; their values are fixed while varying the number of layers.

Figure 2 illustrates the solution of the problem (4) under certain values of the variables $N, R^{I} N, R^{2} N, \ldots, R^{m-}$ ${ }^{l} N$ and the technological coefficients taking random values from the segment $K \subset R^{+}, m=5$. 
a)

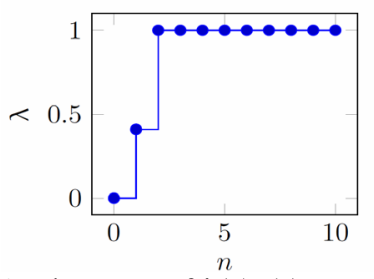

b)

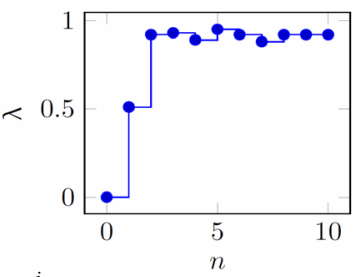

Fig 2. The curve of $\lambda(n)$ : (a) $N=1, R^{i} N=10, i=1, \ldots, 4$,

$$
K=\{1\}, n^{*}=2 \text { and (b) } N=1, R^{i} N=10, i=1, \ldots, 4, K=
$$$$
[1,2], n^{*}=3 \text {. }
$$

Figs. 3 show several solutions of the problem (4), viz., the curves of $\lambda$ under $R^{I} N \in[0,1], R^{2} N \in[0,1]$ with the step $\Delta=0.1$ and the parameter $n$ varying from 1 to 4 .

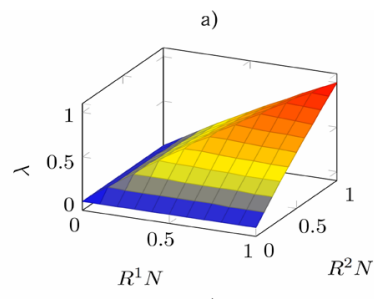

c)

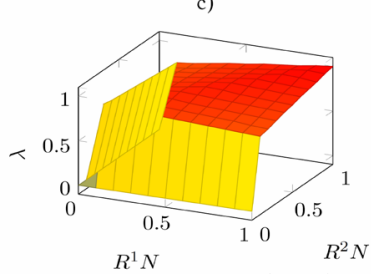

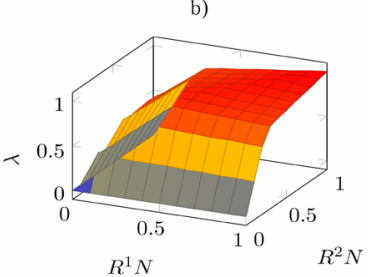

d)

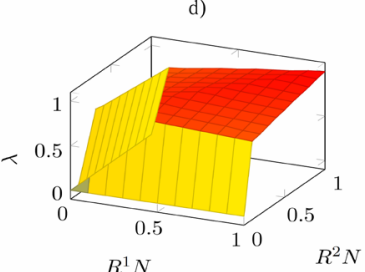

Fig 3. The curve of $\lambda\left(1, R^{l} N: R^{2} N, 1,1\right), K=\{1\}$ :

(a) $n=1$; (b) $n=2$; (c) $n=3$; (d) $n=4$.

\section{Conclusion}

The results of numerical simulation of the structural optimization model for the operating core of an OS have testified to the following:

- considerable reserves of the factors in support facilitiesand a sufficiently high number of STs in all lines of the complete CT allow to achieve the stable maximum of the PF under appreciable deviations in the proportions of the basic production and fluctuations in the technological coefficients of auxiliary production;

- under large reserves of the factors, the increasing variation of the technological coefficients of auxiliary production gets compensated by the growing number of layers in an ST;

- reducing the reserves of the factors and the number of STs of auxiliary production impairs stable production output as the proportions of the basic production deviate from their optimal values.

By incorporating parameters that characterize the internal and external environment of an OS into this model, one can construct complex structural optimization models for the OS Another line of further development of the model concerns designing models of structural dynamics and optimal control. Integration of these two lines of investigations yields control models for the adaptation and development of OSs.

Kharitonov Mikhail has been supported by the Ministry of Education and Science of the Russian Federation (government task No.2.852.2017/4.6).

The study was supported by RFBR, project 16-48-340147r_a.

\section{References}

1. I. Adizes, (Adizes Institute Publications, Los Angeles, 1992)

2. R.R. Blake, J.S. Mouton, (Addison-Wesley Publishing Company, Boston, 1969)

3. J. Champy, (Harper Collins Business, London, 1995)

4. R.M. Grant, S. Rami, R. Krishnan, Sloan management review, 35 (1994)

5. S. Kauffman, (Oxford University Press, New York, 1993)

6. G. Mincberg, (Prentice-Hall, NJ, 1983)

7. P. Watzlawick, J. Weakland, R. Fisch, (Norton Books, New York, 1974)

8. Q. Tran, Y. Tian, American Journal of Industrial and Business Management, 3 (2013)

9. K.E. Weick, R.E. Quinn, Annual Review of Psychology, 50 (1999)

10. R.K. Sah, The American Economic Review, 76, 4 (1986)

11. O. Hart, The Journal of Political Economy, 113 (2005)

12. W.P. Barnett, G.R. Carroll, Annual Review of Sociology, 21 (1995)

13. V.N. Burkov, M.V. Goubko, N.A. Korgin, D.A. Novikov, (Boca Raton: CRC Press, USA, 2015)

14. V.N. Burkov, N.A. Kuznetsov, D.A. Novikov, Automation and Remote Control, 63, 12 (2002)

15. A.A. Voronin, S.P. Mishin, Automation and Remote Control, 63, 5 (2002)

16. A.A. Voronin, S.P. Mishin, Automation and Remote Control, 63, 8 (2002)

17. M.V. Gubko, Automation and Remote Control, 63, 12 (2002)

18. M.V. Gubko, Automation and Remote Control, 70, 1 (2009)

19. M. Kharitonov, A. Svetlov, International Journal of Pure and Applied Mathematics, 107, 4 (2016)

20. A.A. Voronin, M.A. Kharitonov, Automation and Remote Control, 75, 1 (2014)

21. G.B. Kleiner, Finance and Statistics, (1986) 